\title{
Frontogenesis, subduction, and cross-front exchange at upper ocean fronts
}

\author{
Michael A. Spall \\ Department of Physical Oceanography, Woods Hole Oceanographic Institution, Woods Hole, \\ Massachusetts
}

\begin{abstract}
The subduction of water at upper ocean fronts driven by internal instabilities is investigated. A simple analytic model which assumes conservation of potential density and potential vorticity is used to characterize the expected structure of subducted parcels as a function of the surface layer depth and the length scale of the parcel. Parcels subducted from a deep surface layer will be characterized by anomalously low potential vorticity and anticyclonic circulation, while shallow surface layers result in subducted parcels with high potential vorticity and cyclonic circulation. A nonlinear isopycnal primitive equation model is used to demonstrate how baroclinic instability and the resulting frontogenesis force the subduction of parcels below and across the front. Frontogenesis generates vertical velocities of $\mathcal{O}\left(30 \mathrm{~m} \mathrm{~d}^{-1}\right)$ and ageostrophic cross front flows of 3 to $5 \mathrm{~cm} \mathrm{~s}^{-1}$. The subduction is achieved by the deep ageostrophic flow which carries water from the surface layer below and across the front. The subducted parcels quickly develop a circulation through balanced adjustment, anticyclonic for deep surface layers and cyclonic for shallow surface layers. The characteristics of parcels subducted in winter conditions closely resemble a class of eddies called submesoscale coherent vortices. The horizontal and vertical scales of the vortices are determined by the frontogenetic mechanism and the depth of the surface layer. The total permanent subduction rate is estimated to be $20 \mathrm{~m} \mathrm{yr}^{-1}$ for frontal subduction driven by frontogenesis, although local (and temporary) subduction can be much larger. The subducted anticyclonic vortices are often coupled to upper ocean cyclonic vortices, thus forming a baroclinic dipole that propagates at approximately $3.5 \mathrm{~cm} \mathrm{~s}^{-1}$ and is capable of transporting the anomalous water properties far from the region of formation. Similarities with observations in the North Atlantic Subtropical Convergence Zone and near the Gulf Stream are discussed.
\end{abstract}

\section{Introduction}

The process by which water is transferred from the mixed layer into the stratified interior of the ocean is generally referred to as subduction. This process is believed to be important to the physics of both the oceans and the atmosphere. The properties of the main thermocline in the ocean are to a large degree set when the water parcels were last in contact with the atmosphere. Dynamical properties, such as potential vorticity, density, and temperature, play an important role in determining the large-scale circulation and stratification within the main thermocline. The absorption of chemicals from the atmosphere by the ocean depends strongly on the subduction process and may play a key role in the global climate system. In addition, biological activity is particularly large in the near-surface layers, and the exchange of nutrients and biologically active mat-

Copyright 1995 by the American Geophysical Union.

Paper number 94JC02860.

0148-0227/95/94JC-02860\$05.00 ter through subduction can significantly influence the biological cycle [Strass, 1992].

The physics of oceanic subduction are not well known. It is believed to be at least partially controlled by the large-scale variations in atmospheric wind stress curl and net buoyancy flux (see, for example, Marshall et al. [1993, and references therein]). The general pattern of westerlies at midlatitudes and trade winds at low latitudes sets up a convergent Ekman transport with implied downwelling at the region of the subtropical convergence zone where the wind stress curl is a maximum. This vertical velocity contributes at least part of the mass flux from the mixed layer into the permanent thermocline. The surface of the permanent thermocline is not horizontal, however, and a horizontal flow from a region of a deep mixed layer into a region of a shallow mixed layer also transfers mass from the mixed layer into the permanent thermocline. Simple calculations indicate that, on large scales, this mechanism can be of the same order of magnitude as Ekman pumping [Marshall et al., 1993].

Upper ocean fronts are also thought to be places where subduction might occur because they are re- 
gions where deep isopycnals have large vertical excursions and often outcrop into the surface mixed layer. Surface-intensified frontal regions are found in many places throughout the world's oceans. Some are permanent, large-scale features of the general circulation, such as the Gulf Stream, Kuroshio, and the Antarctic Circumpolar Current. Other fronts are intermittent in nature where they may exist for timescales of weeks to months and then break down, such as those found in regions of Ekman convergence like the Subtropical Convergence Zone (STCZ) in the North Atlantic and Pacific. Strong frontal regions are often observed along the periphery of larger-scale mesoscale eddies in the North Atlantic STCZ between $22^{\circ} \mathrm{N}$ and $32^{\circ} \mathrm{N}$ [Halliwell et al., 1991]. Simple scaling estimates by Follows and Marshall [1994] that are based on the ageostrophic axial circulation associated with frontogenesis suggest that subduction driven by frontal processes may be as large as that due to the large-scale Ekman convergence. (Similar ideas are applied to exchange in the atmospheric tropopause by Follows and Austin [1992].) Pollard [1986] found parcels of low potential vorticity water below the mixed layer on the warm side of one of these fronts whose temperature and salinity characteristics suggested that it originated upstream in the mixed layer on the cold side of the front. We focus here on this subclass of fronts which are largely confined to the upper few hundred meters, although many of the results presented here are relevant in a general way to the broader class of surface-intensified fronts. The present focus is motivated by their abundance and the relatively high-resolution data sets which have been collected near these fronts (such as the Frontal Air-Sea Interaction Experiment (FASINEX); see Weller [1991], Pollard and Regier [1992] (hereinafter referred to as PR92), and the data reported by Voorhis and Bruce [1982] (hereinafter referred to as VB82)).

The relative influences of internal frontal processes and external atmospheric forcing on frontal subduction are not known. The evolution of actual upper ocean fronts is likely to be influenced by surface forcing (wind and buoyancy), turbulent mixing in the mixed layer, and large-scale flows in addition to the front's own intrinsic time dependence. In the present study the mechanisms inherent in the interna' instability of the front are isolated from these other complicating factors in order to obtain a clearer understanding of this underlying component. This is justified by the scaling analysis of Follows and Marshall [1994], by the relatively short timescales of baroclinic instability and the associated frontogenesis that are $\mathcal{O}(1$ week $)$, and by the consistency between the results presented here and various observations. It is expected that these results will remain relevant to the more general system, and investigations into the influences of atmospheric forcing and an active mixed layer will be the subject of a later study. Many issues addressed here are also relevant to the more general problem of mass exchange across a potential vorticity gradient.
This paper is outlined as follows. A simple analytical solution that provides some guidelines for the structure and circulation of parcels exchanged across a potential vorticity gradient is presented in section 2 . In section 3 a nonlinear primitive equation model is applied to the problem of frontal subduction for two distinct initial conditions. The subduction mechanism and its relationship to submesoscale coherent vortices are discussed in section 4 , and final conclusions are presented in section 5 .

\section{A Simple Model for Subducted Parcels}

A simple model is developed to describe the structure of parcels that are transferred across a localized potential vorticity gradient. The model is developed and discussed within the context of frontal subduction, however, the underlying dynamics are relevant to the more general class of problems. Frontal regions often mark a transition between different water masses and have an associated change in stratification (or potential vorticity) across the front. This is particularly true for isopycnal layers that outcrop in the frontal region. The thickness of the surface layer is strongly influenced by local atmospheric forcing, however; once subducted below warmer surface layer waters, the water is no longer directly forced by the atmosphere and approximately conserves quantities such as heat and potential vorticity. A consequence of this is that the depth of the surface layer on the cold side of the front is generally different from the thickness of the same isopycnal layer below the surface on the warm side of the front. In the vicinity of mixed layer fronts this transition can take place over horizontal scales of $\mathcal{O}(10 \mathrm{~km})$. If a column of water is transferred from the surface layer to the stratified interior, its thickness will be anomalous relative to the ambient stratification. A schematic of such a subducted water parcel is shown in Figure 1 for the situation where the surface layer is thicker than the same isopycnal layer in the stratified interior. This situation is typical of fronts observed in the North Atlantic STCZ in winter. If the parcel conserves potential vorticity as it is carried across the front, the thermal wind relation requires that the subducted parcel be squashed and spread laterally as it develops a horizontal circulation and negative relative vorticity.

The model consists of three isopycnal layers, as indicated in Figure 1. The thickness of the surface layer on the cold side of the front is $h_{m}$, the thickness of the same isopycnal layer in the interior is $h_{i}$, and the thickness of the subducted parcel is $h$. The horizontal scale of the subducted parcel is $L$, and $g^{\prime}$ is the reduced gravity (assumed to be constant between layers 1 and 2 and layers 2 and 3). Layer 3 is assumed for simplicity to be infinitely thick and motionless. The frontal width and the depth of the surface layer on the warm side of the front are not important, although it is assumed that 


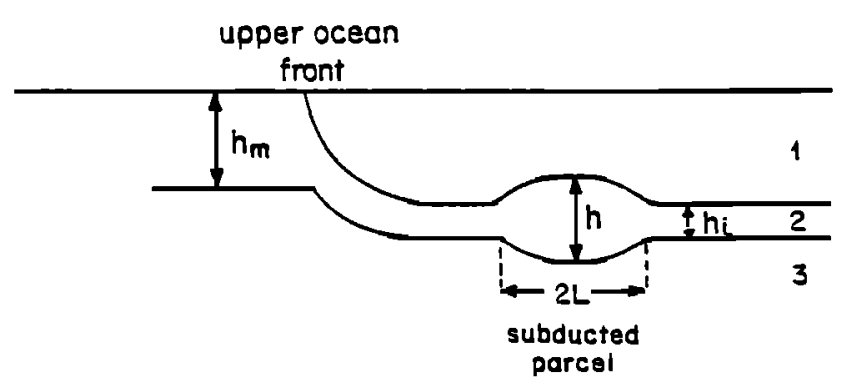

Figure 1. Schematic of the upper ocean front, three isopycnal layer configuration. The surface layer depth is $h_{m}$, the thickness of this isopycnal layer in the stratified interior is $h_{i}$, the thickness of the subducted parcel is $h$, and the radius of subducted parcel is $L$.

the thickness of the upper layer is $\mathcal{O}\left(h_{m}\right)$ and sufficient to prevent outcropping of parcels that are subducted across the front.

We assume that the subducted parcel originated from the cold side of the front in the surface layer with no relative vorticity and that potential density and isopycnal potential vorticity $q$ are conserved as the parcel is subducted below the front. It is, of course, straightforward to modify the potential vorticity to reflect a nonzero initial relative vorticity. The conservation of potential vorticity may be expressed as

$$
q=\frac{f}{h_{m}}=\frac{f+\zeta}{h} .
$$

The planetary vorticity is $f$ (constant), and $\zeta$ is the relative vorticity calculated within an isopycnal layer. By using the isopycnal relative vorticity, we are able to incorporate the twisting terms, which convert horizontal vorticity into vertical vorticity, implicitly into the conservation relation. PR92 have found that these terms may be important in the vicinity of such upper ocean fronts.

For the present analysis we will adopt the somewhat conservative definition of a subducted parcel as one that is carried below the surface layer and away from the front. The physical motivation for this definition is that such parcels are the most likely to be observed in and to impact the general circulation. This approach also allows us to obtain an analytic solution, by assuming that the subducted parcels are characterized by a vortex of radius $L$. Much of the following analysis is qualitatively valid for more general parcel shapes and for parcels which remain dynamically influenced by the front. The mechanism by which the parcel is subducted is not known or specified at this point; however, it will be shown in the following section that frontal instability and frontogenesis can force parcels to cross the front and become subducted in a manner consistent with this simple model.

The velocity within an isopycnal layer is related to the layer thickness through the gradient wind relation as

$$
v=-\frac{f R}{2}+\frac{1}{2}\left(f^{2} R^{2}+4 R g^{\prime} \frac{\partial h}{\partial r}\right)^{1 / 2}
$$

where $R$ is the radius of curvature and $r$ is the radial direction. The relative vorticity is given by

$$
\zeta=\frac{\partial v}{\partial r}+\frac{v}{r} .
$$

The coupled set of (1), (2), and (3) determines the thickness of the isopycnal layer and the circulation velocity of the subducted parcel as a function of radius. Layered approximations to these equations have been solved for by Flierl [1979] (hereinafter referred to as F79), Ikeda [1982], and McWilliams [1988]. Following the procedure of F79, these equations may be combined to form a second-order partial differential equation for the velocity $v$ as

$$
\frac{\partial^{2} v}{\partial r^{2}}+\frac{1}{r} \frac{\partial v}{\partial r}-\frac{1}{r^{2}} v-\frac{v}{L_{d}^{2}}-\frac{v^{2}}{L_{d}^{2} f r}=0
$$

where $L_{d}=\left(g^{\prime} h_{m}\right)^{1 / 2} / f$ is the internal deformation radius. The thickness of the parcel may then be calculated from the velocity as

$$
h=h_{m}\left(1+\frac{1}{f} \frac{\partial v}{\partial r}+\frac{v}{f r}\right) .
$$

The boundary conditions to be satisfied are $v=0$ at $r=0, h=h_{i}$ at $r=L$, where $L$ is the radius of the vortex. Equations (4) and (5) are solved using a fourth-order Runge-Kutta method by integrating from the center of the vortex out to the radius where $h=h_{i}$. The potential vorticity of the vortex (or $f / h_{m}$ ) and the thickness at the center of the vortex are initially specified. The solution then gives the radius of the vortex (or equivalently the Burger number) and the velocity profile. The condition that $h=h_{i}$ at $r=L$ differs from the vanishing layer thickness condition required by F79. This results in a continuous layer thickness, but a discontinuous potential vorticity profile at the edge of the vortex. This is consistent with the idea that the water within the vortex originated on the other side of the front and is thus not constrained (nor expected) to have the same potential vorticity as the surrounding water. It will be shown that this permits a much wider range of both anticyclonic and cyclonic solutions than found in the vanishing layer thickness models of F79, McWilliams [1988], and Killworth [1983].

The thickness of a subducted parcel at the center $h_{0}$ is shown in Figure 2a as a function of the surface layer depth $h_{m}$ and the Burger number $\left(B=g^{\prime} h_{m} h_{i} / f^{2} L^{2}\right)$. All thicknesses have now been nondimensionalized by the interior layer thickness $h_{i}$. Parcels with an anomalous surface layer depth $\left(h_{m} \neq 1\right)$ will develop a circulation once subducted according to (2). The resulting generation of relative vorticity requires a change in thickness in order to conserve potential vorticity as required by (1). For $h_{m}>1$ the subducted parcel will have negative relative vorticity and must thus experi- 

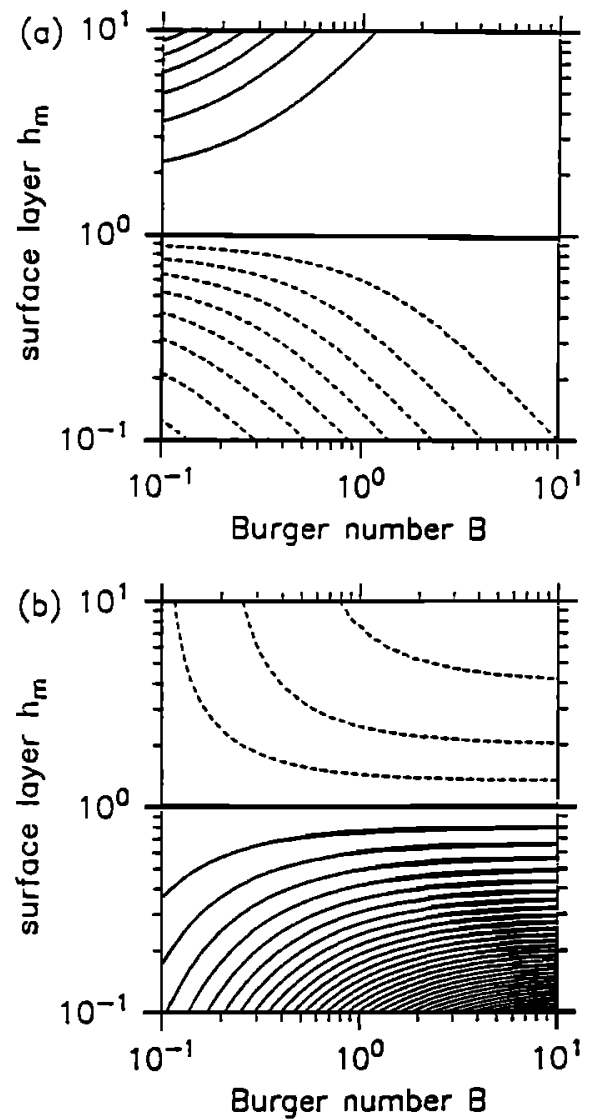

Figure 2. Solution for structure of subducted parcel as a function of initial surface layer depth and the Burger number, (a) parcel thickness (solid lines $h_{0}>1$, contour interval is 1 ; dashed lines $h_{0}<1$, contour interval is 0.1 ), and (b) parcel relative vorticity (solid lines positive, dashed negative, contour interval is 0.25 ).

ence compression, while for $h_{m}<1$ the parcel will have positive vorticity and undergo stretching during subduction. The amount of compression or stretching in the final state is balanced by the offsetting generation of relative vorticity. As expected, if the surface layer is initially deeper than the interior layer thickness, the subducted parcel will also be thicker than the surrounding fluid and thinner than the initial surface layer depth $\left(1<h_{0}<h_{m}\right)$. If the surface layer is the same thickness as the interior, the subducted parcels must also be of the same thickness $\left(h_{m}=h_{0}=1\right)$. A thin surface layer results in local anomalies that are thinner than the surroundings and thicker than the initial surface layer depth $\left(h_{m}<h_{0}<1\right)$. The Burger number is inversely proportional to the horizontal length scale. The subducted parcels maintain a larger portion of their initial surface layer thickness as the horizontal scales become larger (B decreases), while parcels of small horizontal scale must have a thickness that is closer to the ambient thickness. Note that we have made no assumptions about the volume of the subducted parcels. The volume is not constant in Figure 2a; it increases with increasing $h_{m}$ and with decreasing $\mathbf{B}$.
The thickness anomaly of the anticyclones is very small as the radius becomes small $(B \geq \mathcal{O}(1))$ because the maximum slope of the interface is limited by the gradient wind relation. Killworth [1983] showed that for vanishing layer thickness outside the vortex the radius of the vortex cannot be less than $2\left(2 g^{\prime} h_{0}^{*}\right)^{1 / 2} / f$ (where $h_{0}^{*}$ is now the dimensional layer thickness at the vortex center), which means the Burger number of the parcel (based on the parcel thickness) $B=g^{\prime} h_{0}^{*} / f^{2} L^{2}<0.125$. This constraint may be generalized for a Burger number defined with the surface layer thickness and allowing for a nonzero layer thickness outside the vortex to be

$$
B<\frac{h_{m}}{8\left(h_{0}-1\right)}
$$

where $h_{0}$ is the nondimensional thickness of the parcel at the center. This relation is the same as Killworth's when the parcel thickness is large compared to the layer thickness outside the vortex, recovering the previous constraint on the minimum radius of anticyclones. However, anticyclones of smaller radius are permitted when the thickness of the isopycnal layer outside the vortex does not vanish. This smaller scale is consistent with the range of Burger numbers commonly observed in submesoscale coherent vortices [McWilliams, 1985]. The thickness anomaly (and circulation) can be quite large for $B<1$, especially so for vortices with very low potential vorticity (large $h_{m}$ ). In this regime the lines of constant parcel thickness have a slope of $\partial h_{m} / \partial B=8\left(h_{0}-1\right)$, as seen in Figure 2a.

The relative vorticity at the center of the subducted parcel (scaled by $f$ ) is shown in Figure $2 b$ as a function of the surface layer depth and the Burger number. Conservation of potential vorticity requires that all parcels subducted from a surface layer with a depth greater than the internal layer thickness develop anticyclonic vorticity to compensate for vortex compression, while all parcels subducted from shallow surface layers develop cyclonic vorticity. For a surface layer that is just the same thickness as the interior isopycnal layer, the subducted parcel can have no relative vorticity. As the surface layer becomes increasingly deeper (shallower) than the internal layer thickness, the vorticity of the subducted parcel becomes increasingly negative (positive). The gradient wind relation limits the anticyclonic vorticity to be greater than -1 , however, cyclones are not subject to this constraint and can develop very large relative vorticity. The relative vorticity increases with increasing Burger number (as the horizontal length scale decreases) and with increasing thickness anomaly. In the limit of small Burger number the anticyclone thickness $h_{0} \rightarrow h_{m}(1-2 B)$ and the relative vorticity $\zeta \rightarrow-2 B$ become dependent only on the Burger number of the parcel.

\section{Frontogenesis and Subduction}

The simple model discussed in the previous section did not specify the means by which parcels might be 
subducted below the front, nor did the solution determine the length scale of the subducted parcels. It is expected that the vertical velocities will have to be large in order for parcels to be adiabatically subducted near fronts because the isopycnal surfaces are very steep. In addition, we anticipate that some ageostrophic flow component will be required to transfer parcels across the isobars that are coincident with the front. It is now demonstrated that frontogenesis can provide a mechanism for this parcel subduction and scale selection.

Because the theory of frontogenesis is well described in the literature (see, for example, Hoskins [1982]), only a brief review of the mechanism will be included here. Frontogenesis refers to the rapid development of strong horizontal gradients in density from a preexisting weak density gradient. If a confluent large-scale flow field exists, it will act to increase the horizontal density gradient over a portion of the front. The confluent flow might be supplied by a large-scale geostrophic flow field or by finite amplitude baroclinic wave development along a preexisting front. As parcels are carried along the front into the region of confluent flow, they must experience an acceleration in order to remain in cross-front geostrophic balance. This acceleration is compensated for by an ageostrophic velocity according to the semigeostrophic momentum balance as

$$
f v_{a}=\frac{D u_{g}}{D t}
$$

where $v_{a}$ is the ageostrophic cross-front flow and $u_{g}$ is the geostrophic along-front flow. Thus for an upper ocean geostrophic flow that is accelerating downstream, an ageostrophic flow develops directed from the anticyclonic (warm, assuming northern hemisphere) side to the cyclonic (cold) side of the front. As the jet accelerates, cyclonic vorticity is increased on the cyclonic side of the front and anticyclonic vorticity is increased on the anticyclonic side of the front. Conservation of potential vorticity requires that the horizontal flow be convergent on the cold side of the front (downwelling) and divergent on the warm side of the front (upwelling). Mass conservation closes this vertical cell through an ageostrophic flow from the cold side to the warm side in the subsurface layer below the front. A schematic of the familiar ageostrophic circulation in the vicinity of the front is shown in Figure 3 (similar sketches are given by Hoskins [1982], Bleck et al. [1988], and PR92). The deep crossfront ageostrophic flow will tend to carry parcels from the surface layer below and across the front, suggesting that the parcel may become at least temporarily sub-

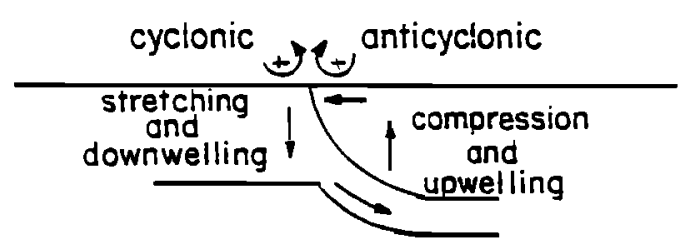

Figure 3. Schematic of ageostrophic velocity resulting from frontogenesis near an upper ocean front. ducted. Wang [1993] has modeled the development of frontogenesis and parcel downwelling in the vicinity of an ocean front and found vertical velocities as large as $80 \mathrm{~m} \mathrm{~d}^{-1}$. This suggested that subduction was taking place, although the model integrations were not long enough to determine the amount or structure of any fluid parcels which might have been permanently subducted across the front. Samelson and Chapman [1995] have modeled the large-amplitude behavior of an upper ocean front similar to case A below using a spectral primitive equation model. They find similar vertical velocities and plumes of low potential vorticity to those reported here.

\subsection{An Isopycnal Primitive Equation Model of Frontal Subduction}

An isopycnal primitive equation model is now used to test whether, under certain circumstances, frontal instability and frontogenesis might lead to parcel subduction, and whether the simple model introduced in the previous section is a useful predictor of the structure of such parcels. We expect primitive equation physics to be necessary to capture the nonlinear aspects of frontogenesis that are important to the development of large Rossby number features and large vertical velocities anticipated to be important in frontal subduction. The isopycnal configuration allows for adiabatic evolution of the flow because cross-isopycnal mixing is not necessary for computational stability. As indicated in section 2, it is expected that this will be important to the final state of the subducted parcels if potential vorticity is conserved. It is recognized that in the real ocean, parcels may not conserve their potential vorticity, particularly in the vicinity of the surface layers, and that the solutions discussed here and in the previous section are most relevant to the ideal limit. I believe that the conservative model is the appropriate place to start because of the clarity and understanding that conservation principles bring to the problem. The results presented here will also provide a basis for understanding the role of nonconservative processes in more complex models in the future.

The model equations. The model used is the isopycnal primitive equation model described by Bleck and Boudra [1986]. The model solves the horizontal momentum equations and the continuity equation within each layer. The layer-integrated horizontal momentum equations are written as

$$
\begin{aligned}
\frac{\partial \mathbf{v}}{\partial t}+ & \nabla \frac{\left|\mathbf{v}^{2}\right|}{2}-(\zeta+f) \mathbf{k} \times \mathbf{v}+\nabla_{\alpha} M \\
& =+(\Delta p)^{-1} \nabla \cdot(A \Delta p \nabla \mathbf{v})+\left(\mathbf{v}^{*}-\mathbf{v}\right) / \gamma
\end{aligned}
$$

where $\mathbf{v}=(u, v)$ is the horizontal velocity vector, $p$ is the pressure, $\mathbf{k}$ is the vertical unit vector, $\zeta=$ $\partial v / \partial x-\partial u / \partial y$ is the relative vorticity, $M=g z+p \alpha$ is the Montgomery potential, $\alpha$ is the specific volume of 
the layer (constant), $\Delta p$ is the pressure thickness of the layer, and $A$ is an eddy viscosity coefficient. An additional term has been added to the standard momentum equations that relaxes $\mathbf{v}$ toward a specified value $\mathbf{v}^{*}$ with time constant $\gamma$. This forcing term is nonzero only over a small downstream extent of the model domain and is used to investigate the influence of a maintained baroclinic inflow of the jet. Features which are advected into this region from upstream (periodic boundary conditions) are slowly suppressed toward the inflow structure specified by $\mathbf{v}^{*}$. The results presented here are qualitatively unchanged without this forcing term; however, after long times (greater than 50 days) the front becomes more diffuse and convoluted and a larger volume of water becomes subducted away from the surface (see section 4).

The continuity equation is represented as a prognostic equation for the layer thickness $\Delta p$,

$$
\frac{\partial \Delta p}{\partial t}+\nabla \cdot(\mathbf{v} \Delta p)=\frac{\Delta p^{*}-\Delta p}{\gamma} .
$$

A restoring term similar to that in the momentum equations is included in the continuity equation to force the layer thickness toward a specified value that is in geostrophic balance with $\mathbf{v}^{*}$.

Initial conditions. The initial conditions reflect a preexisting frontal region that is composed of three isopycnal layers. The front is sufficiently strong that the uppermost layer outcrops in the frontal region, exposing the second layer to the atmosphere on the cold side of the front (indicated schematically in Figure 1). The initial interface depths are defined by the following functions:

$$
\begin{gathered}
p_{1}=\delta_{1} \tanh \left[2\left(x-x_{0}\right) / l\right], \\
p_{2}=D_{2}+\frac{1}{2} \delta_{2}\left(\tanh \left[2\left(x-x_{0}\right) / l\right]-1\right),
\end{gathered}
$$

where $p_{k}$ is the depth of interface $k, \delta_{k}$ is the change in the depth of layer $k$ across the front, $D_{2}$ is the depth of interface 2 away from the front on the warm side, $l$ is the horizontal scale of the front, and $x_{0}$ is the position of the front. For $x<x_{0}$ the interface between layers 1 and 2 outcrops and $p_{1}$ is set to zero. These interface depths also define the forcing terms $\Delta p^{*}$ and $\mathbf{v}^{*}$, with a motionless deep layer. We do not incorporate or study the processes which are responsible for the initial formation of the front or the depth of the surface layers but merely initialize the model with a structure which is consistent with the fronts observed in the STCZ. The body force term may be thought of as a crude parameterization of the actual external forcing which maintains the observed fronts in the ocean.

The model domain is $300 \mathrm{~km}$ by $300 \mathrm{~km}$ with a horizontal grid resolution of $2 \mathrm{~km}$. The boundary conditions are free slip at $x=0$ and $x=300 \mathrm{~km}$ and periodic in the $y$ direction. The Coriolis parameter is taken to be $f=10^{-4} \mathrm{~s}^{-1}$ and constant. The potential density anomalies of the three isopycnal layers are taken to be $25.7,26.0$, and $26.3 \mathrm{~kg} \mathrm{~m}^{-3}$, giving a reduced gravity $g^{\prime}=0.003 \mathrm{~m}^{2} \mathrm{~s}^{-1}$. The body force terms are implemented between $y=0$ and $y=60 \mathrm{~km}$ with a restoring timescale $\gamma=3$ days.

Two cases will be reported here, one with a deep surface layer and one with a shallow surface layer, as shown schematically in Figure 4. The deep surface layer case (case A) used parameter values of $\delta_{1}=100 \mathrm{~m}, \delta_{2}=0 \mathrm{~m}$, and $D_{2}=125 \mathrm{~m}$. In this case the cold surface layer is $125 \mathrm{~m}$ deep and the thickness of this same isopycnal layer away from the front on the warm side is $25 \mathrm{~m}$, a factor of 5 less. The shallow surface layer case (case $B$ ) used values of $\delta_{1}=75 \mathrm{~m}, \delta_{2}=150 \mathrm{~m}, D_{2}=200 \mathrm{~m}$. The shallow cold surface layer is $50 \mathrm{~m}$ deep, while the thickness of this layer away from the front on the warm side is $125 \mathrm{~m}$, a factor of 2.5 more. The cross-front scale is taken to be $l=16 \mathrm{~km}$ in both runs. Instability and meander development are initiated by introducing a superposition of small-amplitude $(1 \mathrm{~km})$ meanders between $75 \mathrm{~km}$ and $300 \mathrm{~km}$ wavelength. The maximum depth of the model domain is $300 \mathrm{~m}$. Additional runs have been carried out with an initially motionless fourth layer between $300 \mathrm{~m}$ and $1000 \mathrm{~m}$ depth with $\sigma_{\theta}=27.3$ that are qualitatively the same as those discussed here, so it is not believed that the shallow model domain significantly influences the results. Turbulent mixing along isopycnal surfaces is parameterized by a Laplacian viscosity with a coefficient of $A=5 \mathrm{~m}^{2} \mathrm{~s}^{-1}$. There is no cross-isopycnal diffusion or stress between layers. The model has been made as inviscid as possible, within numerical stability constraints, in order to use potential vorticity conservation as a guide to the interpretation of the results. While the real ocean may include complex mixing and diabatic effects that are very poorly understood, it is felt that for such a process-oriented study the conceptual advantages of the adiabatic, inviscid limit outweigh the loss of simplicity that must accompany the inclusion of more complex mixing parameterizations.

The deep surface layer front case is similar in parameter space to the fronts in the North Atlantic STCZ studied by VB82, Pollard [1986], Eriksen et al. [1991], PR92, Samelson [1993], and Samelson and Chapman [1995]. It is stressed here that the present calculations

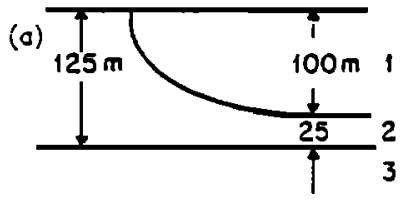

deep mixed layer

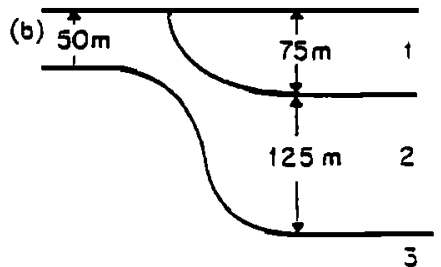

shallow mixed layer

Figure 4. Schematic of model initial conditions for (a) case A, the deep surface layer, and (b) case B, the shallow surface layer. 
are not intended to be a prediction of any particular observed frontal variability. We seek to understand how the internal dynamics of frontal instabilities might lead to the subduction of parcels from the near surface below the front and to characterize this process from two extreme initial conditions, one with a deep surface layer and one with a shallow surface layer.

\subsection{Case A: A Deep Surface Layer}

This experiment is configured to approximate the fronts found in the North Atlantic STCZ in winter, as studied in the Frontal Air-Sea Interaction Experiment (FASINEX) [Weller, 1991, and references therein]. The early evolution of the deep surface layer front is consistent with what is expected based on linear stability theory. The small initial perturbations along the front grow in amplitude through baroclinic instability; that is, the perturbations are gaining energy from the potential energy represented by the sloping isopycnals at the front. The dominant wavelength that emerges from the random perturbations is of the order of $60 \mathrm{~km}$, consistent with the linearly most unstable mode and the observations of VB82. See Samelson [1993] for a discussion of the linear stability analysis of a similar upper ocean front and Samelson and Chapman [1995] for a numerical study of the nonlinear evolution of this most unstable mode. The potential vorticity within layer 2 is shown in Plate $1 \mathrm{a}$ after 8 days of integration. The deep surface layer is characterized by low $q$, while the stratified interior has high $q$ due to its relatively thin layer thickness. The growing instabilities are evident all along the front. The initial relative vorticity within layer 2 is very small, consistent with the assumptions made in the analytic model in section 2.

The front is still evident on day 24 (Plate 1b) as a sharp gradient in potential vorticity, although its path is quite convoluted. Many small-scale features are found near the front, including long filaments, small cyclones and anticyclones, and larger patches of anomalous vorticity. Several regions of low $q$ water have separated on the stratified side of the front. The cores of the vortices have conserved their potential vorticity, although in some cases, water characteristic of the frontal region (intermediate $q$ ) has also been carried away from the front. The isolated vortices are approximately $25 \mathrm{~km}$ in diameter with a very sharp front in potential vorticity separating them from the ambient fluid, consistent with the assumptions made in section 2 . The event that formed the vortex near $x=200 \mathrm{~km}, y=75 \mathrm{~km}$ will be discussed in detail in the next section.

As the parcels are carried across the front into the region of increased stratification, they are also carried down below the warmer surface layer (subducted) and compressed, as anticipated by the simple theory presented in section 2. The thickness of layer 2 is shown in Plate 1c on day 24. Each of the low $q$ parcels has been compressed from its initial thickness of $125 \mathrm{~m}$ in the surface layer to approximately $80 \mathrm{~m}$. Consistent with this compression, each of the subducted parcels has developed anticyclonic vorticity, as indicated in Plate 1d. While the amplitude varies within the vortices, a typical value of the relative vorticity (scaled by $f$ ) is -0.4 . This is sufficiently large that we expect the cyclostrophic force terms to be important in the gradient wind balance. The anticyclonic vorticity and a subsurface maximum in velocity emphasize that these parcels are not simply rings that have pinched off from the frontal instability in a manner consistent with Gulf Stream ring formation events. In fact, these subsurface vorticies appear often to be formed in connection with surface intensified cold core rings, as discussed further in the next section.

The structure and amplitude of the subducted parcels is quite consistent with the results presented in section 2. The nondimensional surface layer depth (parcel thickness before subduction) is $h_{m}=5$, and the subducted parcels have a nondimensional height $h \approx 3.1$ and a relative vorticity at the core of approximately $\zeta=-0.4$. The diameter of the subducted parcels is approximately $25 \mathrm{~km}$, giving a Burger number $B=$ $g^{\prime} h_{m} h_{i} / f^{2} L^{2}=0.24$. The uniform potential vorticity solution discussed in section 2 predicts a nondimensional height of the subducted parcel as $h=2.9$ and the relative vorticity as $\zeta=-0.42$, close to that seen in Plate 1d. This indicates that the structure of the parcels after subduction is not dependent on the details of the subduction process, provided that potential vorticity is conserved during the process. The gradient wind balance is a good approximation to the final dynamical balance of the vortices.

\subsection{Case B: A Shallow Surface Layer}

The early development of the shallow surface layer front (not shown) is similar to that for the deep surface layer front. The development of baroclinic instability and baroclinic waves introduces perturbations to the front that develop cyclonic and anticyclonic vorticity. The timescale of the instability is shorter than for the deep surface layer front because the vertical shear of the velocity is approximately $50 \%$ stronger (maximum initial velocity is $50 \mathrm{~cm} \mathrm{~s}^{-1}$ ) as the interface between layer 2 and 3 now shallows to $50 \mathrm{~m}$ on the cyclonic side of the front. The cyclonic side of the front is now characterized by high $q$ in layer 2 , while the anticyclonic side of the front has relatively low $q$. The frontal instability draws filaments of high $q$ into the low $q$ region, analogous to the process found in the deep surface layer case. Once into the thicker low $q$ water, the subducted plumes are stretched vertically and develop strong cyclonic vorticity.

After 18 days of integration the unstable meanders have produced several small, cyclonic features of high $q$ on the low $q$ side of the front (not shown). The cores of anomalous vorticity are much smaller than the anticyclonic features produced in run A, approximately $8 \mathrm{~km}$ in diameter. This decrease in horizontal scale of the 

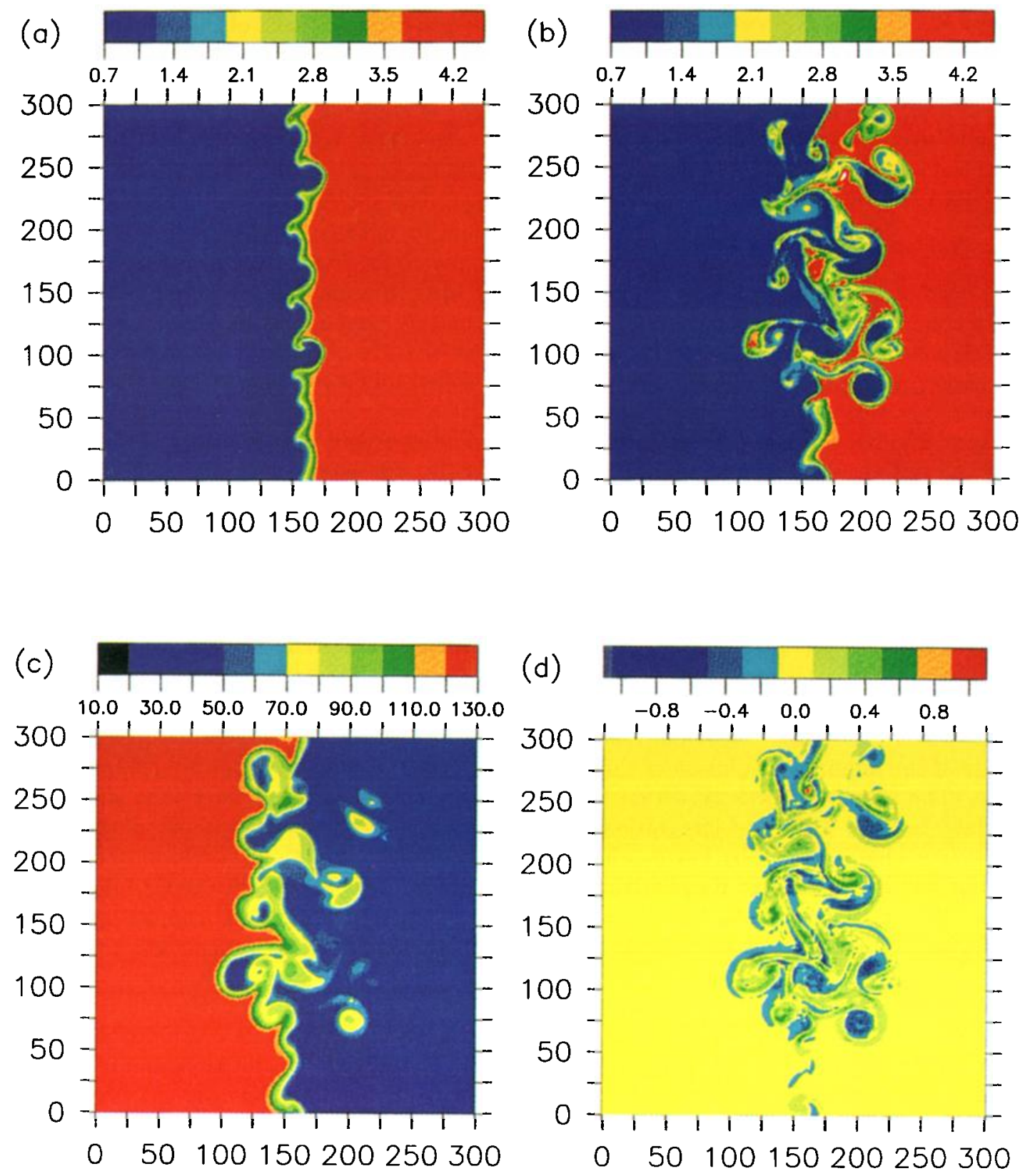

Plate 1. Potential vorticity in isopycnal layer 2 for case A with a deep surface layer. (a) Early meander development on day 8 showing initial protrusion of low potential vorticity water into the stratified interior. (b) Later stage on day 24 , where several patches of low potential vorticity water have crossed under the front and are becoming isolated from the front. (c) Layer thickness and (d) relative vorticity of isopycnal layer 2 on day 24 . Note that subducted low potential vorticity parcels have been compressed and have negative relative vorticity.

core compared to run $A$ is largely because the parcels get stretched vertically when they are subducted (with a compensating horizontal contraction) instead of compression with horizontal spreading. Much of the cyclonic circulation is composed of intermediate $q$ water from beneath the front. The core characteristics of the cyclones are well predicted by the adjustment to gradient wind balance in section 2. For a parcel radius of $4 \mathrm{~km}$ and a surface layer nondimensional thickness of 0.4 , the predicted thickness of the subducted parcels from section 2 is $h=0.81(101.25 \mathrm{~m})$ with a relative vorticity of $\zeta=1.0$, which is in close agreement to that found in the primitive equation model results. This example demonstrates that the qualitative behavior predicted by the simple model is reproduced here and emphasizes the importance of the cross-front potential vorticity gradient for the adjustment process and sense of circulation in the subducted parcels. 


\section{Discussion}

The discussion is focused on the development and dynamics of case A, the deep surface layer, for several reasons. The shallow surface layer is more representative of conditions in summer, when the upper ocean is strongly stratified due to seasonal warming. Parcels that are subducted at a front in summer will probably not be carried out of the seasonal thermocline and are likely to be reentrained into the surface layer in the following winter. Thus while such parcels might be formed and be relevant to the structure of the seasonal thermocline, their influence on the general circulation away from the upper surface layer is likely to be small. In addition, the fronts observed in the STCZ are generally found in winter, when the surface layer is deep relative to the interior stratification.

\subsection{The Subduction Mechanism}

The analysis focuses on the event that formed the anticyclone seen at $x=200 \mathrm{~km}$ and $y=75 \mathrm{~km}$ on day 24 (Plate $1 \mathrm{~b}$ ), although the process is found to be common to all of the subduction events. Long time integrations (100 days) indicate that the same process continues to work at later times, when the front is quite convoluted, but the picture is much clearer for the initial set of formations because of the initially smooth front and quiescent ambient water. The early development of the meander that formed the vortex is typical of a baroclinic wave disturbance on a meandering front. The horizontal velocity is shown superimposed on the potential vorticity in layer 2 on day 8 in Plate $2 a$. The initial conditions had zero velocity in layer 2 and a maximum surface velocity of $35 \mathrm{~cm} \mathrm{~s}^{-1}$. The development of a cyclonic/anticyclonic vortex pair is evident. This situation produces a confluent flow near $x=160 \mathrm{~km}$, $y=55 \mathrm{~km}$, where the flow advects the low $q$ (thick isopycnal layer) toward the region of high $q$ (thin isopycnal layer). This causes an increase in the horizontal gradient of layer thickness (one may think of layer thickness as a proxy for potential temperature) and is conducive to the nonlinear frontogenesis mechanism discussed previously.

The downstream velocity in the upper layer must increase in the region of compressed layer thickness in accordance with the thermal wind relation. As indicated by (7), a cross-stream ageostrophic velocity must balance the geostrophic downstream acceleration in the upper ocean. The resulting convergence in the upper layer leads to a downwelling on the cyclonic side of the front and, through potential vorticity conservation, the development of cyclonic vorticity. The vertical velocity at the interface between layers 2 and 3 is shown in the vicinity of the developing meander on day 12 , superimposed on the potential vorticity in Plate $2 \mathrm{~b}$. The confluence of the layer thickness and, through thermal wind, the acceleration of the upper ocean flow, are in- dicated in the potential vorticity field upstream of the trough. A downward velocity of $30 \mathrm{~m} \mathrm{~d}^{-1}$ is found in the trough and an upward velocity $30 \mathrm{~m} \mathrm{~d}^{-1}$ is found in the crest where the front is advected into the low $q$ water. This stretching and upwelling is a result of the change in ambient layer thickness and drives a strong cyclonic circulation near $x=155 \mathrm{~km}, y=75 \mathrm{~km}$, as indicated by the geostrophic component of the total velocity in Plate 2c. This strong cyclone is consistent with the linear stability predictions of Samelson [1993], the observations of PR92, and the model results of Wang [1993] and Samelson and Chapman [1995]. This strong cyclonic circulation is advecting low $q$ water under the front and high $q$ water out from under the front. Note the beginning development of an anticyclonic circulation along the edge of the cyclonic meander near $x=170 \mathrm{~km}, y=70 \mathrm{~km}$. The ageostrophic velocity is shown superimposed on the potential vorticity field in Plate 2d. There is an ageostrophic flow of approximately $3-4 \mathrm{~cm} \mathrm{~s}^{-1}$ across the potential vorticity front in the same region where anticyclonic vorticity is observed in the geostrophic flow. This cross-front flow is the deep signature of frontogenesis indicated schematically in Figure 3. The ageostrophic flow in the tight cyclone at $x=155 \mathrm{~km}, y=75 \mathrm{~km}$ is in opposite direction to the geostrophic flow, while that along the anticyclonic edge of the developing meander is in the same direction as the geostrophic flow. This is as expected by the gradient wind relation because the geostrophic balance overestimates the flow in cyclones and underestimates the flow in anticyclones.

The ageostrophic velocity advects low $q$ water across the potential vorticity front into the high $q$ stratified interior. The low $q$ water adjusts to the stratified interior according to the gradient wind relation (2). As indicated by the discussion in section 2 , this requires that the low $q$ parcel develop anticyclonic relative vorticity; the early stages of this development are evident in Plate 2c. This adjustment takes place quickly, so the subducted water is developing anticyclonic vorticity even as it is still being carried across the front by the ageostrophic flow. Thus the meander develops a dipole with cyclonic vorticity driven by stretching and anticyclonic vorticity as the low $q$ water is carried across the front and adjusts to the ambient stratification. The dipole pair propagates into the high $q$ region, pulling plumes of low $q$ away from the front as seen in Plate 3a and eventually separates from the front in the high $q$ region. The cyclonic vorticity in layer 2 is suppressed by the compression of the water column, leaving mostly the anticyclone of low $q$ subducted into the region of high $q$. After the parcel has separated from the front, it is approximately $25 \mathrm{~km}$ in diameter and nearly axisymmetric (Plate $1 \mathrm{~b}$ ). By day 40 the dipole pair has propagated well away from the front (Plate $3 \mathrm{~b}$ ), traveling approximately $3.5 \mathrm{~cm} \mathrm{~s}^{-1}$ until it encounters the boundary of the model domain. The adjusted state in layer 2 shows that the anticyclone is dominant over the cyclone in 

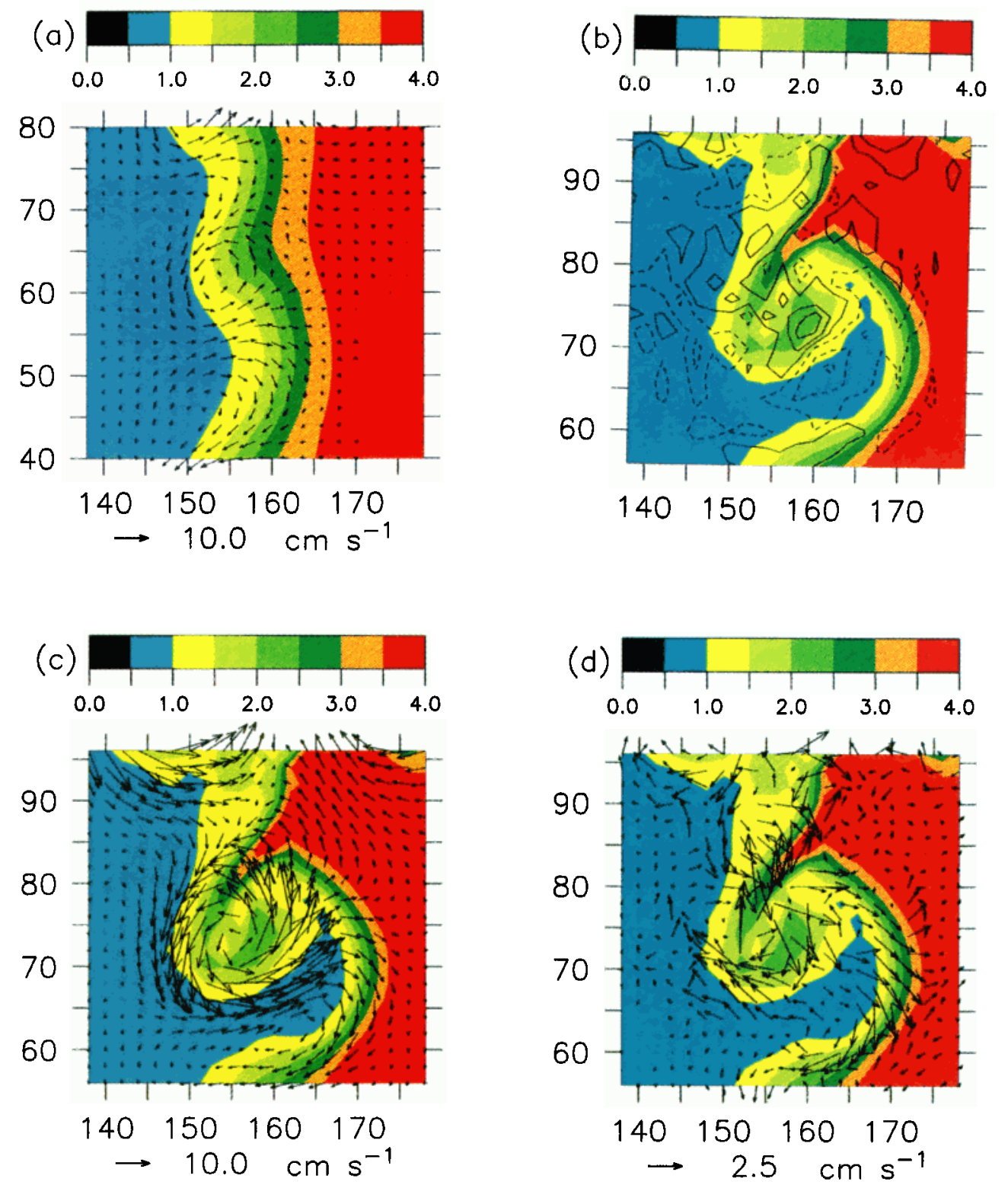

Plate 2. Details of the subduction mechanism for a subregion of case A (deep surface layer). Various components of the velocity field are shown superimposed on the potential vorticity within isopycnal layer 2. (a) Horizonal velocity on day 8 , (b) vertical velocity on day 12 (contour interval is $10 \mathrm{~m} \mathrm{~d}^{-1}$, dashed lines indicate downwelling), (c) geostrophic component of the horizontal velocity on day 12, and (d) ageostrophic component of the horizontal velocity on day 12 .

the deep layer. The maximum geostrophic velocity in layer 2 is approximately $20 \mathrm{~cm} \mathrm{~s}^{-1}$ and the maximum ageostrophic velocity is approximately $5 \mathrm{~cm} \mathrm{~s}^{-1}$ in the same direction as the geostrophic velocity, consistent with the gradient wind balance.

The cyclone is dominant in the upper layer, although there is still some anticyclonic circulation over the deep anticyclone, as shown in Plate 3c on day 40 (the contoured field is now layer thickness). This cyclonic circulation is similar to the more traditional cold ring pinchoff events that are generally associated with vortex formation at fronts. The water in layer 1 with cyclonic vorticity originated within the frontal zone (it has high potential vorticity) and developed its positive relative vorticity as a result of stretching as it was carried away from the front. The water with anticyclonic vorticity in the upper layer did not originate within the front. It has the same low potential vorticity as the surrounding fluid in layer 1 and develops negative relative vorticity as a result of compression due to the deep anticyclone raising the interface between layers 1 and 2 . It is this dipolar pairing of the baroclinic vortices that gives rise to a self-propelling vortex pair that can rapidly carry heat, salt, and other water properties away from the front. Hogg and Stommel [1985] termed such vortex pairs hetons because of their ability to transport heat. 

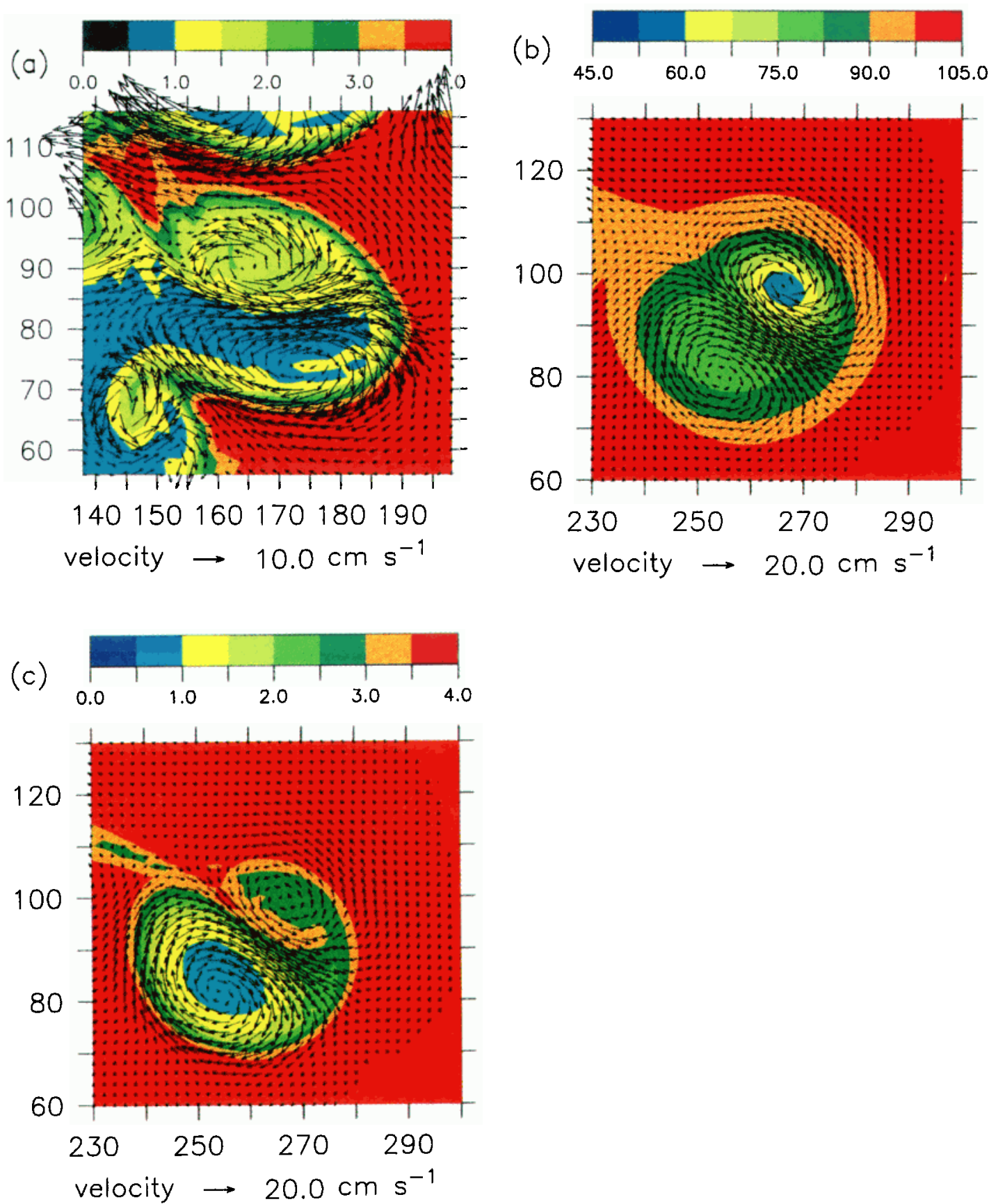

Plate 3. (a) Geostrophic velocity superimposed on the potential vorticity in isopycnal layer 2 on day 18 , (b) total velocity with potential vorticity in layer 2 on day 40 , and (c) total velocity with layer thickness in layer 1 on day 40 .

In some cases a clear dipolar structure does not develop from the model meanders and these subducted anticyclones do not propagate away from the front. They are eventually reentrained by the meandering front and pulled back across (i.e., only temporarily subducted). The presence of a large-scale flow may also serve to advect subducted parcels away from the front and avoid reentrainment, although $\beta$ is likely to become important to the large-scale propagation of these features.

Analogous dipolar structures were found in the quasigeostrophic and primitive equation Gulf Stream mod- els of Robinson et al. [1988] and Spall and Robinson [1990] associated with cold ring formation events. The horizontal scale of the anticyclones in their results was larger than that found here, consistent with the larger deformation radius associated with the Gulf Stream front (approximately $35 \mathrm{~km}$ ) compared with the internal deformation radius for the present upper ocean front (approximately $6 \mathrm{~km}$ ). Wood [1989] also formed deep anticyclones from a primitive equation model of the Gulf Stream and interpreted the sense of circulation through conservation of potential vorticity. Samelson 
and Chapman [1995] find a plume formation similar in the early stages to that discussed here and the formation of a small anticyclone, although they do not find a strong dipole, nor does the anticyclone fully separate from the front in their model. This difference is possibly due to either (1) the presence of cross-isopycnal diffusion in their model or (2) their more continuously stratified vertical representation (spectral decomposition) versus the low-resolution isopycnal representation used here. This is speculation only, however, and further study is required to understand these differences.

\subsection{The Subduction Rate}

A simple estimate of the volume of low potential vorticity water advected across the front by frontogenesis is now obtained. The maximum velocity in layer 1 increases from approximately $40 \mathrm{~cm} \mathrm{~s}^{-1}$ at the crest of the developing meander to approximately $50 \mathrm{~cm} \mathrm{~s}^{-1}$ at the trough as a result of the convergent layer thickness. This acceleration of $10 \mathrm{~cm} \mathrm{~s}^{-1}$ takes place over about $10 \mathrm{~km}$ or in about 0.2 days (following a parcel from crest to trough), giving an acceleration rate of $5 \times 10^{-6} \mathrm{~m} \mathrm{~s}^{-2}$. Together with a Coriolis parameter of $f=10^{-4} \mathrm{~s}^{-1}$, this requires an average ageostrophic cross-front velocity of $5 \mathrm{~cm} \mathrm{~s}^{-1}$ from (7). Let the volume of the subducted parcel be approximated as vol $=2 \pi l^{2} \delta / 3$, where $l$ is the radius of the subducted parcel and $\delta$ is its thickness. This estimate assumes that the height is given by the uniform potential vorticity solution as $h(r)=h\left[1-(r / l)^{2}\right]$. If this ageostrophic flux occurs across the same length scale $l$ along the front, then the time required for the ageostrophic velocity to flux the vortex mass is estimated to be $t=2 \pi l / 3 v_{a}$, where $v_{a}$ is the ageostrophic velocity. For a radius of $l=10 \mathrm{~km}$ and an ageostrophic velocity of $5 \mathrm{~cm} \mathrm{~s}^{-1}$, this gives a formation time of 5 days. This is consistent with the timescale of the modeled and observed frontogenesis events for which the ageostrophic velocity persists.

Keeping in mind the idealized nature of the model, an estimate of the annual subduction rate from the surface layer into the stratified interior driven by frontal instabilities is now made. The total volume of subducted water $V$ is calculated in layer 2 as

$$
V=\frac{\int q h d A-q_{2} \int h d A}{q_{1}-q_{2}}
$$

where $q$ is the potential vorticity, $h$ is the thickness of layer 2 , and the area integral is taken over all of layer 2 where the interface between layer 1 and layer 2 is deeper than $10 \mathrm{~m}$. It is assumed that all of the water in layer 2 is composed of only two types of water, that which originated on the cold side of the front with potential vorticity $q_{1}$ and that which originated on the warm side of the front with potential vorticity $q_{2}$. This will give an upper bound estimate to the subduction rate because water that originated beneath the front with intermediate potential vorticity will contribute to the total subduction volume. This approach is taken in order to avoid arbitrary definitions of "low" potential vorticity water and also to account for subducted water after it has mixed with the ambient water. This estimate includes both the temporary and permanently subducted waters, although the cutoff of $10 \mathrm{~m}$ only influences the estimate of the temporary subduction rate because the permanently subducted water is all much deeper than this depth, typically at depths between $80 \mathrm{~m}$ and $150 \mathrm{~m}$. The volume of permanently subducted water is calculated by performing the integral within layer 2 only at positions greater than $x=200 \mathrm{~km}$, which was chosen to be outside the meander envelope of the front. The subduction rate is then calculated by dividing the total volume of subducted low potential vorticity water by the time of integration and the surface area of the model domain $\left(9 \times 10^{10} \mathrm{~m}^{2}\right)$, assuming that the density of such fronts in the ocean is one per width of the model domain.

The total and permanent subduction rates are given in Figure 5 as a function of time for a 100-day integration. The temporary subduction rate is quite large, between $100 \mathrm{~m} \mathrm{yr}^{-1}$ and $200 \mathrm{~m} \mathrm{yr}^{-1}$ and decreases steadily after day 10. The permanent subduction rate begins to increase on day 10 , as the first vorticies are carried beyond $x=200 \mathrm{~km}$ and after day 25 remains nearly constant at $20 \mathrm{~m} \mathrm{yr}^{-1}$. This indicates that the volume of subducted water increases at a uniform rate and that the subduction mechanism continues to work even after the initially uniform front is strongly convoluted (for example, see Plate 1 for day 24). The size of the subducted parcels later in the calculation are approximately two times larger than those formed early in the calculation because the meander wavelength increases with time, although their characteristics and the formation mechanism appear to be similar. The same calculation was carried out for a model run without the ad hoc body force term in the momentum and continuity equations. This run gives a higher permanent subduction rate of approximately $40 \mathrm{~m} \mathrm{yr}^{-1}$ that is nearly uniform after 50 days. This higher rate is due to the continual relax-

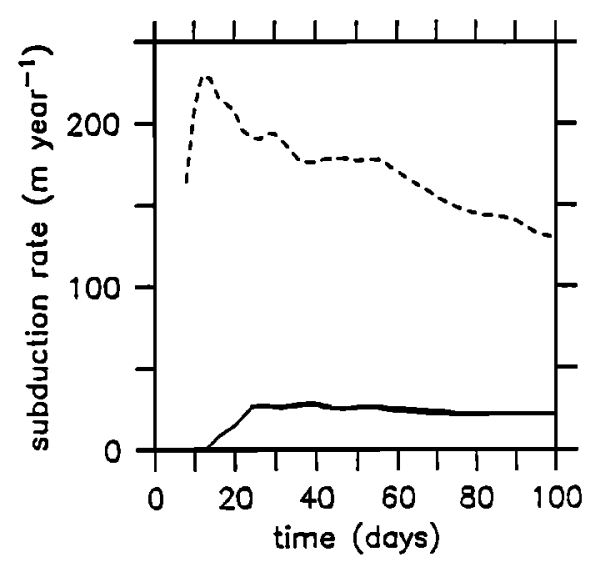

Figure 5. Subduction rate for case A; dashed line is total subduction rate, solid line is the permanent subduction rate. 
ation of the isopycnal slope at the front as a result of baroclinic instability. The addition of the forcing term restores the baroclinicity of the front and inhibits the covering over of water due to the slumping isopycnals. This result suggests that the inclusion of the processes responsible for the initial formation of the front would probably reduce the total permanent subduction, compared with the unforced case, by continually pulling the isopycnals on the cold side of the front up toward the surface.

This subduction rate is less than but of the same order of magnitude as that due to the convergence of the large-scale Ekman transport and variations in the surface layer depth, which is typically $50-100 \mathrm{~m} \mathrm{yr}^{-1}$ at midlatitudes in the subtropical gyre [Marshall et al., 1993]. The present estimate is quite close to the scaling estimate of Follows and Marshall [1994] $\left(25 \mathrm{~m} \mathrm{yr}^{-1}\right)$ and slightly less than the numerical calculations of Samelson and Chapman [1995] ( $37.5 \mathrm{~m} \mathrm{yr}^{-1}$ for the same frontal density as assumed here) for frontal subduction. There are also other differences with the previous estimates, such as the strength of the frontal region (Samelson and Chapman had a stronger front), ambient stratification, model formulation, and time of integration.

4.3. Relationship to Observations in the STCZ, Submesoscale Coherent Vortices, and Deep Anticyclones

The modeled formation mechanism is consistent with several aspects of the observations in the STCZ. Horizontal and vertical ageostrophic flows of similar structure and amplitude to those produced by the model were found by VB82 and PR92, with upwelling and flow toward the front from the warm side in the upper ocean and a corresponding downwelling and flow below the front from the cold side. VB82 described a process of cold water sinking adjacent to the front and across the front in a subsurface layer. These investigators concluded that frontogenesis was taking place; however, they did not directly observe any deep flow to cross the front. The low potential vorticity filament observed under the front by Pollard [1986] suggests that subduction can pull water below the front while conserving its potential vorticity, although the separation of blobs of this water was not observed. The data set of PR92 partially samples a parcel of low potential vorticity water below the surface well away from the front with relative vorticity of approximately $-0.4 f$ and horizontal scale of approximately $10-20 \mathrm{~km}$. The salinity of the blob is much lower than the ambient water and similar to the salinity in the mixed layer on the cold side of the front, suggesting that it might have been formed there in a manner consistent with the present model results. Several features of the plume formation, cyclonic, and anticyclonic vortices are also strikingly similar to observations in the vicinity of shelf break fronts reported by Garvine et al. [1988], although estimates of the vertical circulation in this area were not available.
The properties of parcels subducted by frontogenesis at a deep surface layer front are quite similar to the category of vortices termed submesoscale coherent vortices (SCVs) by McWilliams [1985]. Their characteristic properties may be summarized as follows. They generally have a small vertical scale with a middepth velocity maximum. Their horizontal scales are less than the first deformation radius with a horizontal structure of a single extremum in the geopotential. They tend to be nearly symmetric in the vertical and axisymmetric in the horizontal. Nearly all observed SCVs are anticyclonic with a relative low in the potential vorticity at their center. Although it is clear that many SCVs are of a subsurface origin (e.g., meddies), the properties of others appear to have their origins in the winter mixed layer [Lindstrom and Taft, 1986]. This diversity would seem to suggest that more than one generation mechanism exists. McWilliams [1985] discusses several possibilities and favors strong diapycnal mixing events on small spatial scales and the resulting balanced adjustment, although the processes that control the mixing and determine the horizontal and vertical scales of the anomalous water have not been described.

The low potential vorticity of SCVs suggests that diapycnal mixing is important in setting the initial potential vorticity of the water parcels. The surface layer is a likely place to search for sources due to diapycnal mixing events because mixing is much stronger in the boundary layer than it is in the ocean interior. Boundary mixing is also an important element in the subsurface source proposed by D'Asaro [1988]. The present mechanism does not require that the mixing take place on the submesoscale scales of the vortices, rather it is assumed to take place on the atmospheric synoptic scale, which is much larger than the oceanic cross-frontal scale. The scale of the SCVs is selected through the frontogenesis mechanism acting at upper ocean fronts, which, for the small-scale fronts of the STCZ, forces an ageostrophic flow on scales much less than the first deformation radius of the main thermocline stratification. The large vertical velocity that is coincident with the frontogenesis forces the parcels out of the surface layer and into the permanent thermocline. Large-scale flows or the dipole pairing would then presumably carry the relatively stable vortices away from the source region. The dynamics of the adjusted SCVs is the same as that previously proposed by McWilliams [1988] and Ikeda [1982], namely that of balanced adjustment to an anomalous patch of low potential vorticity water.

Similarities are also noted with larger-scale deep anticyclones found near the Gulf Stream in models [Spall and Robinson, 1990; Robinson et al., 1988] and observations [Richardson, 1993; Bane et al., 1989]. Richardson finds evidence of many deep anticyclones to the south of the Gulf Stream near $700 \mathrm{~m}$ that are approximately as strong as cyclones at this depth. He notes, however, that very few anticyclones are observed in surface 
drifters, suggesting a subsurface maximum in velocity. Bane et al. [1989] document one such anticyclone and find a radius of $150-200 \mathrm{~km}$ with deep maximum swirl velocities of greater than $50 \mathrm{~cm} \mathrm{~s}^{-1}$ at $900 \mathrm{~m} \mathrm{depth}$ and surface velocities approximately $25 \mathrm{~cm} \mathrm{~s}^{-1}$. This increased horizontal scale of the anticyclones is consistent with the scale found in the models of Robinson et al. [1988] and Spall and Robinson [1990] and as expected for the larger deformation radius and meander scale in the vicinity of the Gulf Stream compared with the fronts of the STCZ. Richardson [1993] also documents the apparent formation of a deep anticyclone from the upstream side of a meander as a cold ring was formed. This is just the time and location that the frontogenesis mechanism proposed here forms the deep anticyclones.

\section{Conclusions}

The process of subduction from the upper ocean below and across a preexisting upper ocean front in the absence of surface forcing has been investigated using both a simple analytic model and an isopycnal primitive equation model. The fronts studied here are roughly modeled after the surface-intensified fronts observed in the Subtropical Convergence Zone of the North Atlantic, although many of the conclusions may be relevant to a wider variety of fronts and to the general problem of mass exchange across a potential vorticity gradient. It has been shown that baroclinic wave growth along the front can trigger regions of frontogenesis, which are characterized by periods of large vertical velocities of $\mathcal{O}\left(30 \mathrm{~m} \mathrm{~d}^{-1}\right)$ and cross-front ageostrophic flows of $3-5 \mathrm{~cm} \mathrm{~s}^{-1}$, consistent with observations and previous models. The downwelling on the cold side of the front pulls water down and below the front, at least temporarily subducting it from the ocean surface. Only that portion of the anomalous water that is carried across the front by the ageostrophic deep circulation can be permanently subducted, however, and the remainder of the plume returns to the cold side of the front. The subducted water has anomalous potential vorticity, relative to the ambient fluid, and develops a circulation through balanced adjustment to the gradient wind relation, consistent with the analytic model. Deep anticyclonic circulations are often formed in connection with the more traditional surface-intensified cyclonic cold core rings as a dipole pair that quickly propagates away from the front, carrying with it anomalous water properties from the opposite side of the front.

A total subduction rate driven by frontogenesis of approximately $20 \mathrm{~m} \mathrm{yr}^{-1}$ is estimated. This is somewhat less than estimates of subduction due to large-scale variations in atmospheric forcing, which are typically 50$100 \mathrm{~m} \mathrm{yr}^{-1}$. The present estimate is rough, however, given the uncertainties in the number of fronts in the ocean, the neglect of external forcing, and the absence of an active mixed layer. Temporary (or local) subduc- tion rates are typically much larger, between 100 and $200 \mathrm{~m} \mathrm{yr}^{-1}$. The internal processes of frontogenesis and subduction are very important to the local dynamics of upper ocean fronts and contribute significantly to their local variability. They are also likely to be very important to the local biological activity and exchange between the properties of the turbulent mixed layer and the permanent thermocline.

The structure of parcels subducted from a deep surface layer closely resembles a class of vortices called submesoscale coherent vortices. Their characteristic features (small horizontal scales, subsurface maximum, anticyclonic vorticity) are consistent with the subduction mechanism discussed here. The volume of the vortices is determined by the space and timescales of the frontogenesis, typically producing anticyclonic vortices of approximately $25 \mathrm{~km}$ in diameter over timescales of 1 week. The vertical scale of the vortices is determined by the depth of the surface layer, relative to the subsurface stratification. Parcels that are subducted in late winter would be the most likely to be observed in the permanent thermocline because their signal would be large and they would most easily escape reentrainment into the deepening mixed layer in the following winter. This suggests that those submesoscale vortices whose temperature and salinity characteristics identify their origin in the winter mixed layer may have been formed through frontal subduction. Similarities with deep anticyclones observed south of the Gulf Stream are also noted.

Acknowledgments. Support for this work was provided by the Office of Naval Research grants N00014-90-J-1490 and N00014-93-1-0572. I would like to thank Roger Samelson and Dave Chapman for helpful and interesting discussions and also for sharing their preliminary results. Peter Killworth made helpful suggestions regarding the analytic models in section 2. Comments and criticisms by two anonymous reviewers also led to an improved presentation of the results. This is contribution $\mathbf{8 7 3 3}$ from the Woods Hole Oceanographic Institution.

\section{References}

Bane, J. M., L. M. O'Keefe, and D. R. Watts, Mesoscale eddies and submesoscale, coherent vortices: Their existence near and interactions with the Gulf Stream, in Mesoscale/Synoptic Coherent Structures in Geophysical Turbulence, edited by J. Nihoul and B. Jamart, pp. 501518, Elsevier, New York, 1989.

Bleck, R., and D. Boudra, Wind-driven spin-up in eddyresolving ocean models formulated in isopycnic and isobaric coordinates, J. Geophys. Res., 91, 7611-7621, 1986.

Bleck, R., R. Onken, and J. D. Woods, A two-dimensional model of mesoscale frontogenesis in the ocean, $Q . J . R$. Meteorol. Soc., 114, 347-371, 1988.

D'Asaro, E., Generation of submesoscale vortices: A new mechanism, J. Geophys. Res., 93, 6685-6693, 1988.

Eriksen, C. C., R. A. Weller, D. L. Rudnick, R. T. Pollard, and L. A. Regier, Ocean frontal variability in the Frontal 
Air-Sea Interaction Experiment, J. Geophys. Res., 96, 8569-8592, 1991.

Flierl, G. R., A simple model for the structure of warm and cold core rings, J. Geophys. Res., 84, 781-785, 1979.

Follows, M̄. J., and J. F. Áustin, Á zonaì average model of the stratospheric contributions to tropospheric ozone, J. Geophys. Res., 97, 18,047-18,060, 1992.

Follows, M. J., and J. C. Marshall, Eddy driven exchange at ocean fronts, Ocean Modell., 102, Hooke Inst. Oxford Univ., Oxford, England, pp. 5-9 + 6 figures, 1994.

Garvine, R. W., K.-C. Wong, G. G. Gawarkiewicz, and R. K. McCarthy, The morphology of shelfbreak eddies, J. Geophys. Res., 93, 15,593-15,607, 1988.

Halliwell, G. R., P. Cornillon, K. H. Brink, R. T. Pollard, D. L. Evans, L. A. Regier, J. M. Toole, and R. W. Schmitt, Descriptive oceanography during the Frontal Air-Sea Interaction Experiment: Medium- to large scale variability, J. Geophys. Res., 96, 8553-8568, 1991.

Hogg, N. G., and H. M. Stommel, The heton, an elementary interaction between discrete baroclinic geostrophic vortices, and its implications concerning eddy heat-flow, Proc. R. Soc. London A, 397, 1-20, 1985.

Hoskins, B. J., The mathematical theory of frontogenesis, Annu. Rev. Fluid Mech., 14, 131-151, 1982.

Ikeda, M., A simple model of subsurface mesoscale eddies, J. Geophys. Res., 87, 7925-7931, 1982.

Killworth, P. D., On the motion of isolated lenses on a betaplane, J. Phys. Oceanogr., 13, 368-376, 1983.

Lindstrom, E. J., and B. A. Taft, Small water property transporting eddies: An analysis of statistical outliers in the Local Dynamics Experiment hydrographic data, J. Phys. Oceanogr., 16, 613-631, 1986.

Marshall, J. C., A. J. G. Nurser, and R. G. Williams, Inferring the subduction rate and period over the North Atlantic, J. Phys. Oceanogr., 23, 1315-1329, 1993.

McWilliams, J. C., Submesoscale coherent vortices in the ocean, Rev. Geophys., 23, 165-182, 1985.

McWilliams, J. C., Vortex generation through balanced adjustment, J. Phys. Oceanogr., 18, 1178-1192, 1988.

Pollard, R. T., Frontal surveys with a towed profiling conductivity/temperature/depth measurement package (SeaSoar), Nature, 323, 433-435, 1986.
Pollard, R. T., and L. A. Regier, Vorticity and vertical circulation at an ocean front, J. Phys. Oceanogr., 22, 609-625, 1992.

Richardson, P. L., A census of eddies observed in North Atlantic SOFAR float data, Prog. Oceanogr., 31, 1-50, 1993.

Robinson, A. R., M. A. Spall, and N. Pinardi, Gulf Stream simulations and the dynamics of ring and meander processes, J. Phys. Oceanogr., 18, 1811-1853, 1988.

Samelson, R. M., Linear instability of a mixed layer front, J. Geophys. Res., 98, 10,195-10,204, 1993.

Samelson, R. M., and D. C. Chapman, Evolution of the instability of a mixed layer front, J. Geophys. Res., in press, 1995.

Spall, M. A., and A. R. Robinson, Regional primitive equation studies of the Gulf Stream meander and ring formation region, J. Phys. Oceanogr., 20, 985-1016, 1990.

Strass, V. H., Chlorophyll patchiness caused by mesoscale upwelling at fronts, Deep Sea Res., Part A, 39, 75-96, 1992.

Voorhis, A. D., and J. G. Bruce, Small-scale surface stirring and frontogenesis in the subtropical convergence of the western North Atlantic, J. Mar. Res., 40, S801-S821, 1982.

Wang, D. P., Model of frontogenesis: Subduction and upwelling, J. Mar. Res., 51, 497-513, 1993.

Weller, R. A., Overview of the Frontal Air-Sea Interaction Experiment (FASINEX): A study of air-sea interaction in a region of strong oceanic gradients, J. Geophys. Res., 96, 8501-8516, 1991.

Wood, R. A., Eddy generation by instability of a highly ageostrophic front: Mean flow interactions and potential vorticity dynamics, in Mesoscale/Synoptic Coherent Structures in Geophysical Turbulence, edited by J. Nihoul and B. Jamart, pp. 265-278, Elsevier, New York, 1989.

M. A. Spall, Department of Physical Oceanography, Woods Hole Oceanographic Institution, Woods Hole, MA 02543. (e-mail: spall@cms.whoi.edu)

(Received April 4, 1994; revised October 12, 1994; accepted November 1,1994 .) 